\title{
Elevated Body Swing Test: A New Behavioral Parameter for Rats with 6-Hydroxydopamine-Induced Hemiparkinsonism
}

\author{
Cesario V. Borlongan and Paul R. Sanberg \\ Division of Neurological Surgery, Departments of Surgery, Neurology, Psychiatry, and Pharmacology, \\ University of South Florida College of Medicine, Tampa, Florida 33612
}

Parkinson's disease is characterized by a depletion of dopamine (DA) neurons in the nigrostriatal pathway. Stereotaxic injections of 6-hydroxydopamine (6-OHDA), a selective neurotoxin, into either the medial forebrain bundle or the substantia nigra result in a massive DA denervation of the nigrostriatal pathway. Following unilateral nigrostriatal DA depletion, hemiparkinsonian animals develop a stereotypical rotational behavior when challenged with DA agonists such as apomorphine. The drug-induced rotational behavior has been widely used as the behavioral index of hemiparkinsonian animals, but it has some limitations. Although asymmetries in the rotational behavior may indicate an imbalance of DA contents and release capacity in the bilateral nigrostriatal pathway, the behavior is a pharmacological reaction. Accordingly, the drug-induced rotation test is subject to sensitization effects. The present study proposes the elevated body swing test (EBST) as a measure of asymmetrical motor behavior of hemiparkinsonian animals in a drug-free state. The EBST simply involves elevating the animal by handling its tail and recording the frequency and direction of the swing behavior. Unilateral nigral 6-OHDA-lesioned rats exhibited significant biased swing activity with the direction contralateral to the lesioned side, corresponding to the direction of apomorphine-induced rotations. A 30 sec EBST was noted as the peak time for biased swing activity. At $7 \mathrm{~d}$ postlesion (the start of testing), and every week thereafter for a period of 2 months, a fairly stable biased swing activity level was observed. At 1 and 2 months postlesion, the same animals were also challenged with apomorphine. High positive correlations between swing and apomorphine-induced rotational behavior were noted. Furthermore, tail pinch or apomorphine injection increased the level of biased swing activity in the lesioned animals. Similar mechanisms implicated in the dopamine-mediated rotational behavior may be involved in the swing behavior. The EBST may circumvent the problem of sensitization and pose as an alternative tool in characterizing spontaneous behavior in animals with lesions of the nigrostriatal pathway.

Received Jan. 23, 1995; revised Jan. 23, 1995; accepted Mar. 3, 1995.

This work was supported in part by Theracell, Inc., Menlo Park, CA, and Dr. David W. Cahill, Division of Neurological Surgery Funds. We thank Timothy Randall for excellent technical assistance in the behavioral tests.

Correspondence should be addressed to Dr. Cesario V. Borlongan, Division of Neurological Surgery, Department of Surgery, University of South Florida, College of Medicine, 12901 Bruce B. Downs Boulevard, MDC Box 16, Tampa, FL. 33612 .

Copyright $@ 1995$ Society for Neuroscience $0270-6474 / 95 / 155372-07 \$ 05.00 / 0$
[Key words: basal ganglia, 6-hydroxydopamine, Parkinson's disease, apomorphine, sensitization, rotational behavior, motor behavior asymmetry]

Animal models of Parkinson's disease usually involve damaging the nigrostriatal dopaminergic pathway, which has been demonstrated to resemble the neuropathology of the disease (Bernheimer et al., 1973; McGeer et al., 1977). 6-Hydroxydopamine (6-OHDA) is the widely used selective neurotoxin for inducing massive dopamine (DA) denervation of the nigrostriatal pathway in animals (Ungerstedt and Arbuthnott, 1970; Creese and Iversen, 1974). Unilateral stereotaxic injections of 6-OHDA into either the medial forebrain bundle or the substantia nigra destroy dopaminergic neurons on one side of the brain, thus creating a unilateral lesion of the nigrostriatal pathway (Ungerstedt, 1971a,b; Creese and Snyder, 1979). These lesioned animals are known to develop drug-induced stereotypical rotational behavior (Ungerstedt, 1971a,b; Silverman and Ho, 1981; Coward, 1983). Administration of apomorphine activates the supersensitive receptors on the lesioned side of the brain, causing the animal to rotate selectively in the direction contralateral to the lesioned side. In contrast, administration of amphetamine stimulates the release of DA from neurons on the intact side of the brain, inducing the animal to turn in the direction ipsilateral to the lesioned side.

Rotational behavior of unilateral 6-OHDA-lesioned animals in response to DA receptor agonists is the conventional method in assessing dopamine-mediated responses (Norman et al., 1990; Hudson et al., 1993b). However, sensitization due to repeated drug administration may confound interpretation of the druginduced rotational behavior (Bevan, 1983; Coward, 1983; Kalivas and Weber, 1988), especially when assessing the efficacy of neural transplants (Norman et al., 1990). For example, in transplanted animals with hemiparkinsonism, a normalization of the drug-induced behavior may indicate a recovery from imbalance in DA contents and releasing capacity in the bilateral nigrostriatal pathway, but the ubserved behavior is a pharmacological reaction (Hattori et al., 1992).

Chronic use of the majority of stimulants (e.g., amphetamines) may result in the development of greater sensitivity to the drugs' effects (Kuczenski and Leith, 1981; Segal and Kuczenski, 1987a,b; Masur et al., 1986; Stewart and Vezina, 1988; Phillips et al., 1994). Of note, the neurochemical system implicated with mediation of drug-induced stimulation is the mesolimbic-striatal DA system (Dworkin and Smith, 1987; Wise, 1988; Phillips et al., 1994). It is spccifically pointed out that the DA release and reuptake mediate the behavioral sensitization to amphetamine 

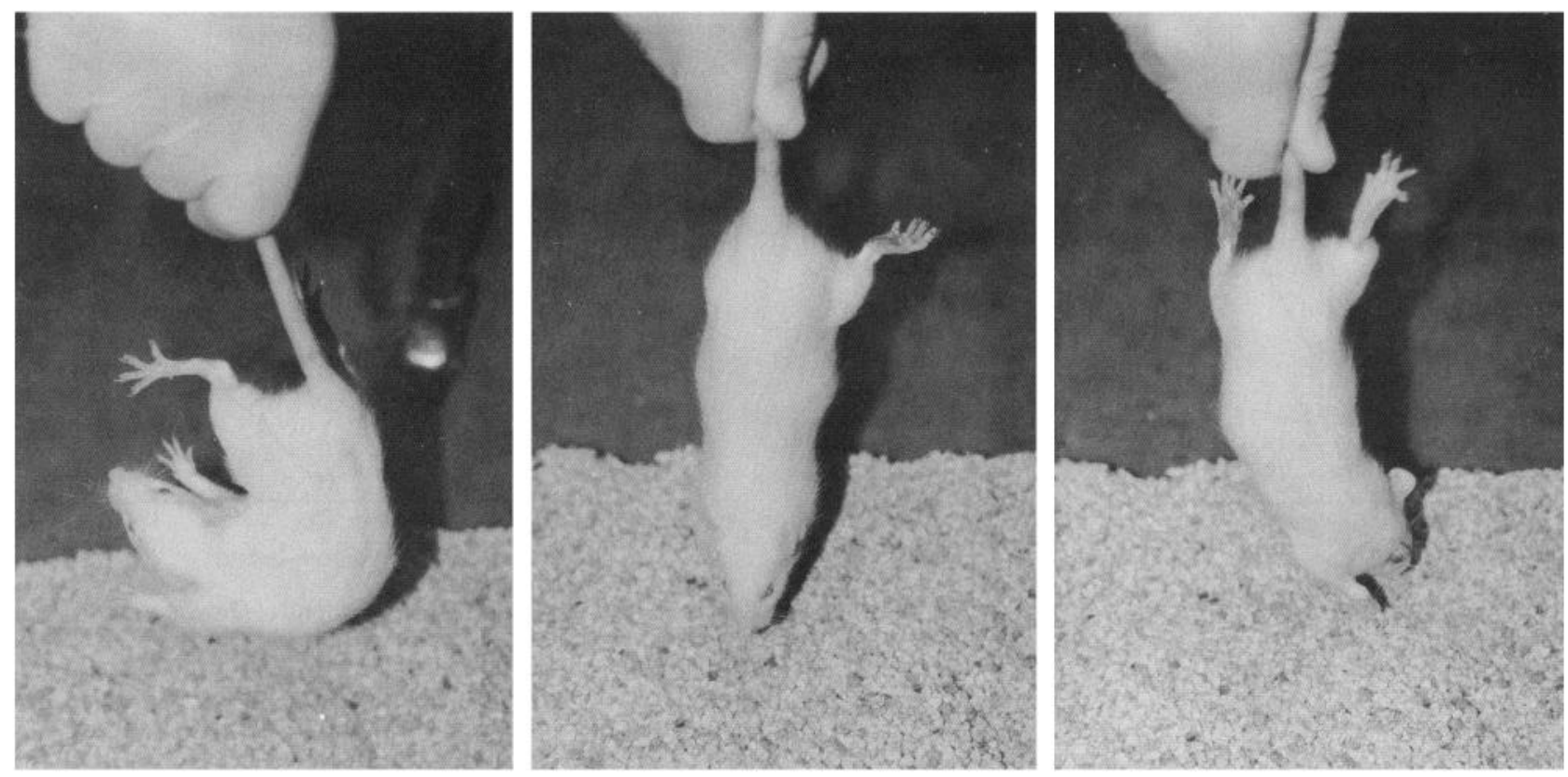

Figure 1. Vertical axis (middle). The animal is shown positioned at the vertical axis, which was defined as no more than $10^{\circ}$ to either left or right side. The animal was held approximately at 1 inch from the base of its tail. The height between the animal and the surface was about 1 inch. Right-biased swing (left). The animal is shown at the right-biased position. A right swing was counted when the head of the animal moved more than $10^{\circ}$ from the vertical axis to the right side. Left-biased swing (right). The animal is shown at the left-biased position. A left swing was counted when the head of the animal moved more than $10^{\circ}$ from the vertical axis to the left side.

(Kalivas and Weber, 1988; Robinson et al., 1988; Phillips et al., 1994).

Similar behavioral sensitization effects have been observed in chronic administration of apomorphine. Repeated apomorphine injections lead to progressively greater increases in locomotor activity (Mattingly et al., 1991; Rowlett et al., 1991). Unilateral nigral 6-OHDA-lesioned rats were found to exhibit a significant increase in rotations during the second injection of apomorphine (Norman et al., 1990), which was quite robust in one study allowing a 5-6 week interval between injections (Klug and Norman, 1993).

These studies would preclude occurrence of sensitization to drugs that induce rotational behavior in unilateral 6-OHDA-lesioned animals. Thus, a behavioral parameter that can evaluate 6-OHDA-lesioned animals in a drug-free state may reflect a more natural response of the animals to the lesion effects. The present study was conducted to evaluate the feasibility of elevated body swing test (EBST) as a measure of asymmetrical motor behavior in hemiparkinsonian animals tested in a drugfree state. The EBST involves measurement of frequency and direction of the swing behavior of the animal when it is held by the tail. It is hypothesized that an animal with unilateral nigral 6-OHDA lesions will exhibit biased swings, with the direction (contralateral to the lesioned side) the same as that of the apomorphine-induced rotations.

\section{Materials and Methods}

Animals. Male, 8-week-old Sprague-Dawley rats were obtained from Harlan Sprague-Dawley, Inc., Indianapolis, IN. Animals were housed in pairs with free access to food and water on a $12 \mathrm{~L}: 12 \mathrm{D}$ cycle. Initially, baseline data for swing and apomorphine-induced rotations were obtained. Only animals $(n=64)$ not showing any biased behavior were included in the study.

Surgical procedures. Forty animals were randomly selected and stereotaxically lesioned in the left substantia nigra (AP $-5.0, \mathrm{ML}+1.5$,
DV -8.0 ) with $8 \mathrm{mg}$ of 6-OHDA (Sigma Chemical Co.) in $4 \mathrm{ml} 0.9 \%$ saline containing $0.02 \%$ ascorbic acid. Rats were first anesthetized with sodium pentobarbital $(60 \mathrm{mg} / \mathrm{kg}$, i.p.) and mounted in a Kopf stereotaxic frame. The 6-OHDA solution was injected over a 4 min period and the needle left in place for an additional $5 \mathrm{~min}$ before retraction. The remaining 24 animals, which served as controls, underwent sham lesions. These animals were introduced to the same surgical procedures but saline, instead of 6-OHDA, was injected.

Behavioral testing. The swing test is a simple and easy behavioral test that only requires handling the animal by its tail and recording the direction of swings made by the animal for a certain period of time. Initially, the animal was placed into a Plexiglas box $(40 \times 40 \times 35.5$ $\mathrm{cm}$ ), allowed to habituate for $2 \mathrm{~min}$ and attain a neutral position, defined as having all four paws on the ground. The animal was held approximately 1 inch from the base of its tail. It was then elevated to an inch above the surface on which it has been resting (Fig. 1). The animal was held in the vertical axis, defined as no more than $10^{\circ}$ to either the left or the right side. A swing was recorded whenever the animal moved its head out of the vertical axis to either side. Before attempting another swing, the animal must return to the vertical position for the next swing to be counted. In cases when the animal swung and redoubled its efforts to move toward one side without returning to the vertical position, only one swing was counted. In a few cases when the animal refused to return to the vertical position for more than $5 \mathrm{sec}$ or when it grabbed its tail, time was stopped and the animal was momentarily placed back on the ground. Once in a neutral position, the animal was then resuspended and time was restarted. Swings were usually exhibited in less than $1 \mathrm{sec}$, thus frequency, and not amount of time, of swings was counted. When the animal did not commence swing behavior when it was elevated for more than $5 \mathrm{sec}$, a gentle pinch to the tail induced the behavior. Swings were recorded using a hand counter. The total number of swings made to each side was divided by the overall total number of swings made to both sides to get percentages of left and right swings. The criterion for biased swing behavior was set at $70 \%$ or higher.

EBST over $60 \mathrm{sec}$. At $7 \mathrm{~d}$ postlesion, 16 rats comprising of 8 6-OHDA-lesioned rats and 8 sham-lesioned rats, were subjected to EBST for $60 \mathrm{sec}$. To show time points of biased swing activity, swing responses were counted over four consecutive $15 \mathrm{sec}$ segments. Biased swing activity for each segment was computed. Two observers who were blind to the lesion status of the animals simultaneously recorded 


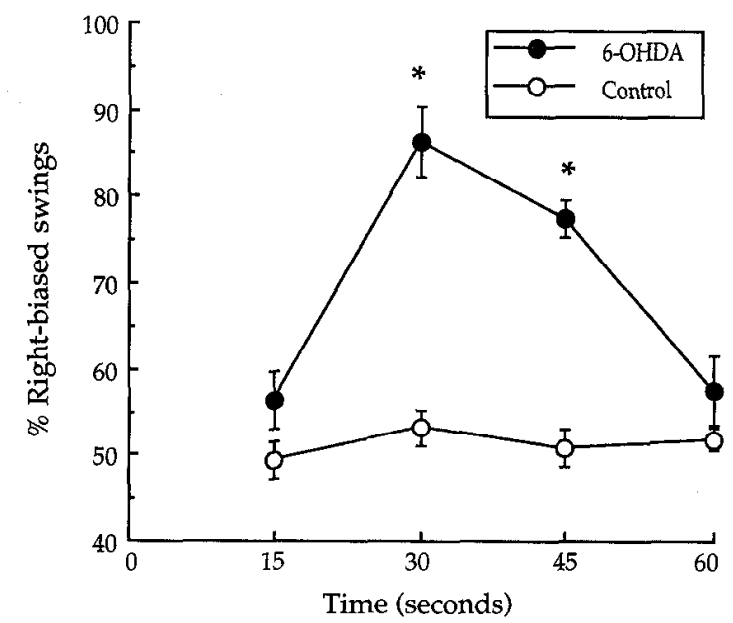

Figure 2. EBST over $60 \mathrm{sec}$. At 30 and $45 \mathrm{sec}$, 6-OHDA-lesioned animals exhibited right-biased swings of $70 \%$ or higher compared to normal rats. Asterisks $\left({ }^{*}\right)$ indicate significant mean differences at $\alpha=$ 0.01 .

the number of swing responses. Biased swing scores recorded by the two observers were compared for interobserver agreement.

Repeated EBSTS over 2 months. A new set of 16 animals (8 6-OHDA- and 8 sham-lesioned rats) were introduced to the EBST once a week, starting at $7 \mathrm{~d}$ postlesion, for 8 consecutive weeks, and once at $84 \mathrm{~d}$ postlesion. The EBSTs were conducted for $30 \mathrm{sec}$. Tests were conducted blind.

Correlation between EBST and 6-OHDA. To test the correlation between the swing and DA agonist-induced rotational behavior, the same animals used above (repeated EBSTs over 2 months) were injected with apomorphine at 1 month and 2 months postlesion. These 2 apomorphine-induced rotation tests were conducted to obtain a more accurate measure of correlation with the swing behavior. Animals were first introduced to the EBST prior to the rotation test. At least an hour interval separated the two tests. For the apomorphine-induced rotation test, the animal was allowed to habituate for $2 \mathrm{~min}$ in a Plexiglas box ( $40 \times 40$ $\times 35.5 \mathrm{~cm}$ ), and then injected intraperitoneally with $0.20 \mathrm{mg} / \mathrm{kg}$ apomorphine (Sigma Chemical Co.) to induce rotations. Immediately after the injection, the number and direction of rotations were recorded for 30 min using a Rotoscan monitor (Omnitech Electronics, Inc., Columbus, $\mathrm{OH}$ ). The data used for subsequent analyses comprised of the net full-body $\left(360^{\circ}\right.$ rotation) contralateral turns minus the total ipsilateral turns.

Possible mechanisms underlying EBST. At approximately 2 months postlesion, new sets of animals were introduced to the EBST with minor modifications. In one additional test designed to investigate stressor effects, the investigator pinched the tails of the animals $(n=8)$ throughout the duration of the swing test. In the other test designed to study the role of the dopaminergic system, the animals $(n=8)$ were injected intraperitoneally with apomorphine $(0.20 \mathrm{mg} / \mathrm{kg})$ just immediately prior to commencing the EBST. Eight sham-lesioned animals were also introduced to the tail pinch- and apomorphine-induced EBST, and eight other 6-OHDA-lesioned animals were introduced to the conventional EBST either with or without saline injection. All tests were conducted blind.

Statistical analysis. For the swing behavior, a two-way ANOVA was used to analyze swing behavior data across the $15 \mathrm{sec}$ segments, and also across the 2 month period. Post hoc tests were then carried out using Tukey HSD (honestly significant difference) test. Pearson $r$ correlation was used to assess interobserver agreement. For the tail pinchand the apomorphine-induced EBST, differences in swing behavior data were analyzed using the multigroup ANOVA and Tukey HSD test. For the drug-induced rotational behavior, separate analyses of data from the 1 month and the 2 months tests were done using Student's $t$ tests. Pearson $r$ correlation was again used to evaluate correlation between swing behavior and rotational behavior.

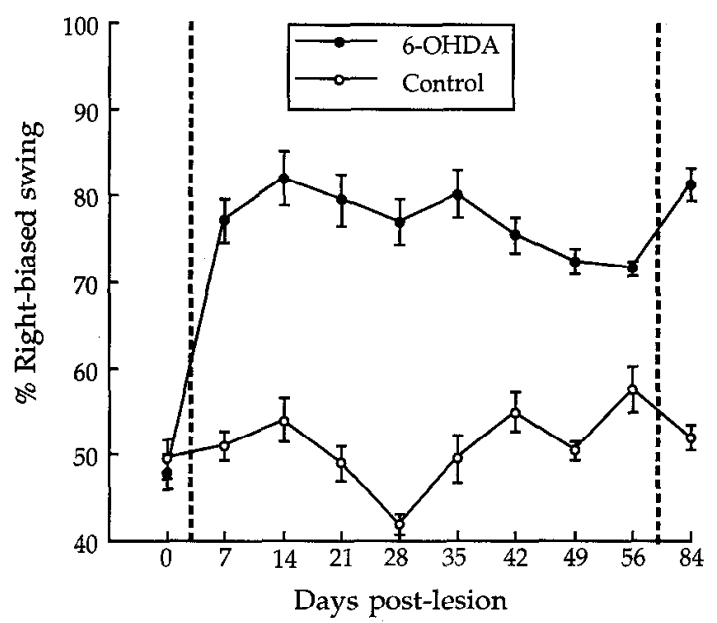

Figure 3. Repeated EBSTs over 2 months. Only animals showing unbiased swings (prelesion) were used in the study. 6-OHDA-lesioned animals exhibited significant right-biased swings throughout the entire postlesion test period, but note a slight decline at 42,49 , and $56 \mathrm{~d}$ postlesion. A restored high level of biased activity, however, was observed after the 1 month rest period ( $84 \mathrm{~d}$ postlesion). Similar high levels of biased swing activity were noted when animals were subjected to tail pinch or apomorphine challenge. Sham-lesioned animals did not display any biased swing on all test sessions.

\section{Results}

EBST over $60 \mathrm{sec}$

ANOVA revealed an overall significant $F$ ratio $[F(1,14)=$ 51.88, $p<0.001]$. Across the $15 \mathrm{sec}$ segments, we observed that 6-OHDA-lesioned animals exhibited $70 \%$ or higher number of contralateral (to the lesion) biased swings at the segments 15 to $30 \mathrm{sec}$ and 30 to $45 \mathrm{sec}$ (mean differences 33.00 and 26.75, HSD $=9.85, \alpha=0.01$ ). Accordingly, subsequent EBSTs were conducted for $30 \mathrm{sec}$ duration. Interobserver data revealed Pearson $r$ correlations of 0.95 or higher between scores of the two observers across the four segments. These high correlations were noted for both 6-OHDA-lesioned and normal rats.

\section{Repeated EBSTs over 2 months}

Figure 3 shows the percentages of swing responses exhibited by both nigral 6-OHDA- and sham-lesioned rats. 6-OHDA-lesioned animals exhibited significant right-biased swing behavior, which persisted from $7 \mathrm{~d}$ postlesion, the earliest time the EBST was conducted, and each week thereafter up to 2 months postlesion. Sham-lesioned animals did not display any biased swing behavior throughout the experiment. The observed mean biased swing activities displayed by the 6-OHDA-lesioned animals were $70 \%$ or higher, with the lowest being $71.6 \%$ and the highest being $82 \%$. ANOVA revealed that 6-OHDA-lesioned animals exhibited a significant biased swing activity across the postlesion test periods $[F(1,14)=20.06, p<0.001]$. Comparisons of the two groups of animals for each testing day revealed significant right-biased swing responses in 6-OHDA-lesioned animals in all testing days. All mean differences were greater than the highest HSD value of $8.51(\alpha \mathrm{s}=0.01)$. However, there was a slight trend of decreasing biased swing activity during the sessions 42 , 49 , and $56 \mathrm{~d}$ postlesion. A month following the last swing test ( $84 \mathrm{~d}$ postlesion), the lesioned rats exhibited a high level of biased swing activity, which was significantly higher than the biascd swing levcl at $56 \mathrm{~d}$ postlesion (mean difference exceeds $\operatorname{HSD}=4.38, \alpha=0.01$ ). 


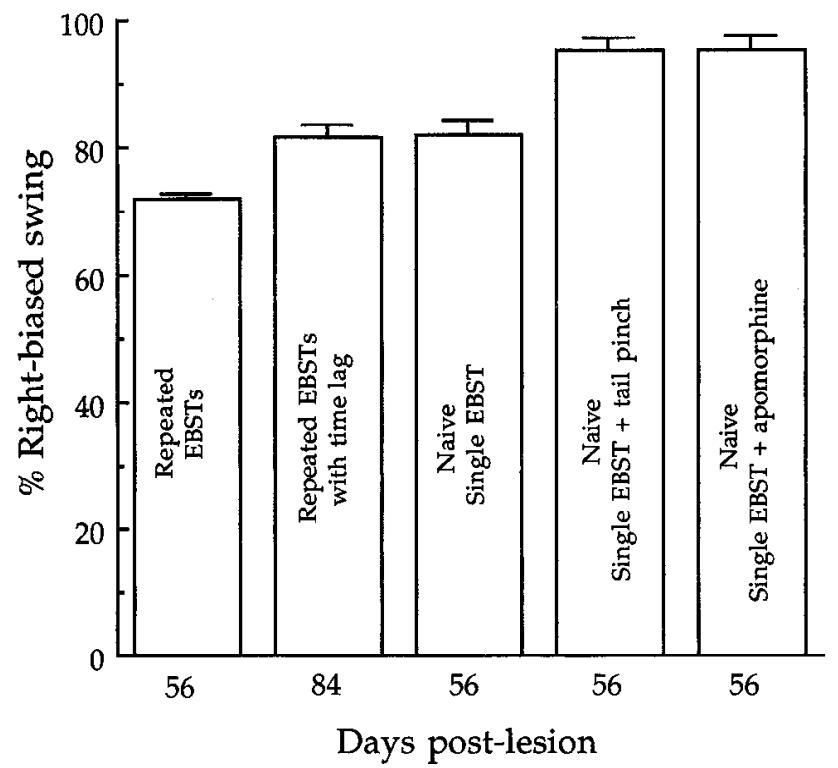

Figure 4. Tail pinch- and apomorphine-induced biased swing behavior. Each bar corresponds to mean \pm SEM biased swing activity level at $56 \mathrm{~d}$ postlesion of 6-OHDA-lesioned animals not previously exposed (naive) to EBST. Data from animals repeatedly tested in EBST (56 and $84 \mathrm{~d}$ postlesion) are also presented to better show comparisons of biased swing activity of animals subjected to tail pinch or injected with apomorphine. Naive untreated animals had a significantly higher biased swing level than repeatedly EBST tested animals at $56 \mathrm{~d}$ postlesion (mean difference $=10.00, \mathrm{HSD}=9.69, \alpha=0.01$ ), but did not differ significantly from the repeatedly tested animals at $84 \mathrm{~d}$ postlesion. Tail pinch-induced biased swing activity level was also found to be significantly higher than either the biased swing level of animals singly or repeatedly tested in EBST at $56 \mathrm{~d}$ postlcsion (mean differences $=13.12$ and $23.12, \alpha=0.01$ ) or animals tested at $84 \mathrm{~d}$ postlesion (mean difference $=13.44, \alpha=0.01$ ). Apomorphine-induced biased swing activity level was similarly noted to be significantly higher than the two groups of repeatedly tested animals $(\alpha=0.01)$.

\section{Correlation between EBST and 6-OHDA}

6-OHDA-lesioned rats exhibited significant rotations contralateral to the side of the lesion compared to sham-lesioned rats at 1 month $[t(14)=12.01, p<0.01]$ and 2 months postlesion $[t(14)=18.61, p<0.01]$. Since 6-OHDA-lesioned rats received lesions on their left side, the contralateral turns corresponded to clockwise rotations. Thus, these apomorphine-induced rotations appear to demonstrate the same side of biased swing activities observed in 6-OHDA-lesioned animals. Indeed, analyses of data from swing behavior and apomorphine-induced rotations revealed high positive correlations (Fig. 4). Pearson $r \mathrm{~s}$ of 0.85 and 0.80 were recorded at 1 month and 2 months postlesion, respectively, in 6-OHDA-lesioned animals. In contrast, no significant correlations between swing behavior and rotational behavior were found in sham-lesioned animals.

\section{Possible mechanisms underlying EBST}

All naive 6-OHDA-lesioned animals exhibited $70 \%$ or higher contralateral biased swing compared to sham-lesioned animals at $56 \mathrm{~d}$ postlesion (Fig. 5). An overall significant $F$ ratio was obtained $[F(2,21)=15.99, p<0.001]$. Naive 6-OHDA-lesioned animals either subjected to saline injection or not prior to EBST did not differ significantly; therefore, data from these two groups were combined. 6-OHDA-lesioned animals showed a higher percent of right-biased swing activity when their tails were pinched or when systemically challenged with apomor-

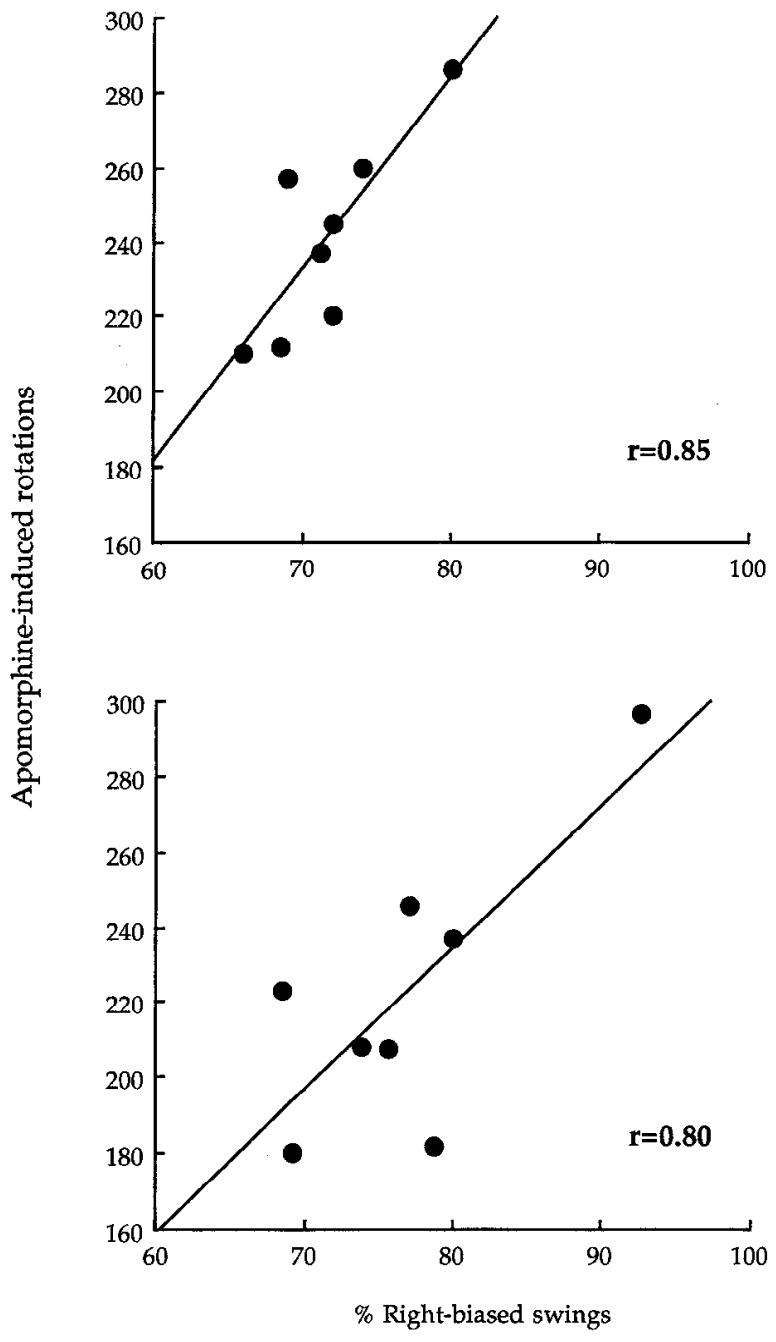

Figure 5. Correlations between swing and apomorphine-induced rotations for 6-OHDA-lesioned animals. Upper graph represents the 1 month postlesion data, while the lower graph corresponds to the 2 month postlesion data. Pearson $r \mathrm{~s}$ of 0.85 and 0.80 were noted at 1 month and 2 months postlesion, respectively.

phine compared to naive 6-OHDA-lesioned animals tested in the conventional EBST (mean differences exceed HSD $=11.30$, $\alpha \mathrm{s}=0.01$ ), indicating the effects of stress and dopamine levels, respectively. Both tail pinch and apomorphine treatments resulted in biased swing activity levels of $95 \%$; hence, no significant difference was observed between these treatments.

Biased swing activity levels of the three groups of naive animals were then compared with the data obtained at 56 and 84 $\mathrm{d}$ postlesion from the repeatedly tested 6-OHDA-lesioned animals. The overall $F$ ratio $[F(4,35)=26.75, p<0.01]$ was statistically significant. The naive untreated animals had a signiticantly higher biased swing level than repeatedly EBST-tested animals at $56 \mathrm{~d}$ postlesion (mean difference $=10.00$, IISD $=$ $9.69, \alpha=0.01$ ), but did not differ significantly from the repeatedly tested animals that had a time lag of 1 month prior to the next EBST ( $84 \mathrm{~d}$ postlesion). These results indicate that handling did affect the level of biased swing activity. Tail pinchinduced biased swing activity level was also found to be significantly higher than either the biased swing level of animals singly or repeatedly tested in EBST at $56 \mathrm{~d}$ postlesion (mean differences $=13.12$ and $23.12, \alpha=0.01$ ) or animals tested at 84 
$\mathrm{d}$ postlesion (mean difference $=13.44, \alpha=0.01$ ), demonstrating the effectiveness of this treatment to produce a higher level of biased swing activity. Apomorphine-induced biased swing activity level was similarly noted to be significantly higher than the two groups of repeatedly tested animals $(\alpha=0.01)$, providing evidence that apomorphine magnified existing imbalance in the dopaminergic system that seems to underlie the biased swing behavior.

\section{Discussion}

The present study demonstrated that rats with 6-OHDA lesions in the nigrostriatal pathway displayed a biased swing activity that correlated highly with the conventional apomorphine-induced rotational behavior. Employing the EBST at early periods postlesion also revealed the sensitivity of this test, like the rotation test, to the behavioral deficits consequent to unilateral nigrostriatal DA depletion. Most studies using hemiparkinsonian rats have reported the onset of DA agonist-induced rotational behavior at around 2 to 4 weeks postlesion, but some studies have noted rotational behaviors as early as a few days immediately following 6-OHDA lesions (Ungerstedt, 1971a; Staunton et al., 1981). We also observed similar biased swing activity (C. V. Borlongan and P. R. Sanberg, unpublished observations) upon awakening of the animals following the stereotaxic surgery. However, we have some misgivings on such early timing of behavioral testing since surgical procedures (cannula or vehicleinduced transient disruption of the pathway) may confound the true neurotoxin lesion effects (Waddington and Crow, 1979).

The objective of the study was to the circumvent the sensitization effects inherent in repeated measures of behavioral function using the drug-induced rotation tests. Of note, we observed no sensitization effects at the 2-month apomorphine-induced rotation test. Although other studies have reported a sensitization effect after a single injection of apomorphine (doses of $1.0 \mathrm{mg} /$ $\mathrm{kg}$ and higher), the 1 month interval between our two apomorphine injections may have prevented the development of this sensitization effect. Past studies support these effects of time lag between injections and number of drug injections on sensitization (Martres et al., 1977; Bevan, 1983; Coward, 1983; Morelli and Di Chiara, 1987; Mattingly et al., 1988a, b; Mattingly and Rowlett, 1989). Alternatively, congruent with studies reporting experiential facilitation of sensitization (Morelli and Di Chiara, 1987; Mattingly and Gotsick, 1989; Mattingly et al., 1991), the EBST may have interfered with the onset of the sensitization effect. Further studies incorporating repeated apomorphine-induced rotation tests with repeated EBSTs are warranted to investigate possible EBST's effects on the development of the sensitization effect.

Similar behavioral tests with 6-OHDA-lesioned animals in a drug-free state have been reported. Using a treadmill apparatus to measure motor function of 6-OHDA-lesioned rats, animals were induced to run uphill on the treadmill by electrostimulation (Hattori et al., 1992). Although this method is free from confounding effects of sensitization to drugs, sensitivity to electrical stimulation becomes the apparent problem. Another technique suggested is an automated recording of open-field locomotor activities (Sanberg et al., 1987; Hudson et al., 1993a). Although this technique ensures quantification of a variety of locomotor variables, the cost of the activity monitors seems expensive. A more common drug-free test is the paw-reaching test, which involves measurement of the skilled reaching of the paw contralateral to the lesioned side (Dunnett et al., 1988; Abrous et al., 1992). The concern here is the inherent subjective scaling ( 0 , weak; 1 , moderate; 2 , exaggerated) and the complexity of the paw-reaching task. In addition, the paw reaching task and apomorphine-induced rotations are negatively correlated, which might imply that different types of behaviors are mediated by the dopaminergic pathway. Another drug-free behavioral test that is also negatively correlated to the rotational behavior employs recording of asymmetrical orientation to edges of an openfield (Sullivan et al., 1994). In this test, undrugged 6-OHDAlesioned animals were found to preferentially align with the edge of a platform with the intact striatum contralateral to the edge. The negative correlation between edge and rotational behavior may be due to the former behavior being a sensorimotor response and the latter behavior being a motor response (Sullivan et al., 1994). The swing behavior was noted to be positively correlated with the rotational behavior, and may be considered a motor response. Recently, Schallert and colleagues (1994) also described a preferential use of the ipsilateral (to the lesion) forelimb for postural and exploratory movements in 6-OHDA-le sioned animals, which supports our observation that the ipsilateral forelimb was consistently used in making the contralateral swing. Further characterization of the swing behavior may reveal interesting correlations with those behaviors reported by Schallert and colleagues.

The possible mechanism involved in the display of biased swing behavior may be the stress caused by handling the animal by its tail. Past studies have reported that tail pinch and stressor effects may result in changes in locomotor activity (Boutelle et al., 1990; Tanaka et al., 1991; Rouge-Pont et al., 1993). Furthermore, stressors may interact with the dopaminergic pathway (Pei et al., 1990; Cenci et al., 1992). An imbalance then in DA levels between the two sides of the brain, coupled with stressor effects, may explain the observed biased swing activity. Neurochemical studies on tail pinch and stressors have revealed increases in DA metabolite, DOPAC, and ${ }^{3} \mathrm{H}-\mathrm{SCH} 23390$ binding (Morelli et al., 1987; Rodriguez and Castro, 1991; Rowlett et al., 1991, 1993). Of note, a higher level of biased swing activity was observed when the animal's tail was pinched or when the animal was injected with apomorphine. These results, taken together, would suggest that the swing activity is a dopaminemediated motor response. A similar mechanism is also implicated in apomorphine-induced rotational behavior (Ungerstedt, 1971a,b; Silverman and Ho, 1981; Coward, 1983). Future investigations into the neurochemical alterations during swing activity of animals with lesions in the dopaminergic pathway may reveal further evidence of interaction between DA and stressor effects. One may argue though that if swing behavior is a stress-mediated function, then the saline injection should have increased the swing activity level. However, the "stress" associated with the saline injection might have diminished already during the EBST session, compared with a continuous stress delivered by the tail pinch.

In EBST sessions toward the end of the 2 month period, the 6-OHDA-lesioned animals displayed a decreasing trend of biased swing activity. This could be the result of lower stress levels due to repeated handling. However, the 2 month weekly test period seems to be a sufficient time to evaluate any behavioral altcrations following brain insults. Furthermore, animals showed a restored high level of biased swing activity when tested 1 month following the last swing test ( $84 \mathrm{~d}$ postlesion) or when their tails were pinched.

In summary, the EBST has been shown to be a rapid, easy, 
inexpensive and accurate measure of a dopamine-mediated motor function. The biased swing behavior was shown to be a good estimate of true effects of unilateral 6-OHDA lesions on the nigrostriatal pathway. Repeated behavioral assessment is very important when evaluating effects of neural transplants on rat models with hemiparkinsunism. The EBST may circumvent the sensitization problem, and pose as an alternative tool for studies of animal models of neurodegenerative disorders characterized by asymmetrical brain lesions.

\section{References}

Abrous DN, Wareham AT, Torres EM, Dunnett, SB (1992) Unilateral dopamine lesions in neonatal, weanling and adult rats: comparison of rotation and reaching deficits. Behav Brain Res 51:67-75.

Bernheimer HW, Birkmayer W, Hornykiewicz O, Jellinger K, Seitelberger F (1973) Brain dopamine and the syndromes of Parkinson and Huntington. Clinical, morphological and neurochemical correlations. J Neurol Sci 20:415-455.

Bevan P (1983) Repeated apomorphine treatment causes behavioral supersensitivity and dopamine $\mathrm{D}_{2}$ receptor hyposensitivity. Neurosci Lett 35:185-189.

Boutelle MG, Zetterstrom T, Pei Q, Svensson L, Fillenz M (1990) In vivo neurochemical effects of tail pinch. J Neurosci Methods 34:151157.

Cenci MA, Kalen P, Mandel RJ, Bjorklund A (1992) Regional differences in the regulation of dopamine and noradrenal release in the medial frontal cortex, nucleus accumbens and caudate-putamen: a microdialysis study in the rat. Brain Res 581:217-228.

Coward DM (1983) Apomorphine-induced circling behavior in 6-hydroxydopamine-lesioned rats. Arch Pharmacol 323:49-53.

Creese I, Snyder SH (1979) Nigrostriatal lesions enhance striatal $\left[{ }^{3} \mathrm{H}\right]$ apomorphine and $\left[{ }^{3} \mathrm{H}\right]$ spiroperidol binding. Eur J Pharmacol 56: 277-281

Dunnett SB, Isacson O, Sirinathsinghji DJ, Clarke DJ, Bjorklund A (1988) Striatal grafts in rats with unilateral neostriatal lesions---III. Recovery from dopamine-dependent motor asymmetry and deficits in skilled paw reaching. Neuroscience 24:813-820.

Dworkin SI, Smith JE (1987) Neurobiological aspects of drug-seeking behaviors. In: Neurobehavioral pharmacology, Vol 6, Advances in behavioral pharmacology (Thompson T, Dews PB, Barrett JE, eds), pp 1-43. Hillsdale, NJ: Erlbaum.

Hattori S, Li Q, Matsui N, Nishino H (1993) Treadmill running test for evaluating locomotor activity after 6-OHDA lesions and dopaminergic cell grafts in the rat. Brain Res Bull 31:433-435.

Hudson JL, Levin DR, Hoffer BJ (1993a) A 16-channel automated rotometer system for reliable measurement of turning behavior in 6-hydroxydopamine lesioned and transplanted rats. Cell Transplant 2:507-514.

Hudson JL, Van Horne CG, Stromberg I, Brock S, Clayton J, Masserano J, Hoffer BJ, Gerhardt GA (1993b) Correlation of apomorphine- and amphetamine-induced turning with nigrostriatal dopamine content in unilateral 6-hydroxydopamine lesioned rats. Brain Res 626:167-174.

Kalivas PW, Weber B (1988) Amphetamine injection into the ventral mesencephalon sensitizes rats to peripheral amphetamine and cocaine. J Pharmacol Exp Ther 245: 1095-1 102.

Klug JM, Norman AB (1993) Long-term sensitization of apomorphineinduced rotation behavior in rats with dopamine deafferentation or excitotoxin lesions of the striatum. Pharmacol Biochem Behav 46: 397-403.

Kuczenski R, Leith NJ (1981) Chronic amphetamine: is dopamine a link in or a mediator of the development of tolerance and reverse tolerance? Pharmacol Biochem Behav 15:405-413.

Martres MP, Costentin J, Baudry M, Marcais H, Protais P, Schwartz JC (1977) Long term changes in the sensitivity of pre- and postsynaptic dopamine receptors in mouse striatum evidenced by behavioral and biochemical studies. Brain Res 136:319-337.

Masur J, Oliveira de Souza ML, Zwicker AP (1986) The excitatory effect of ethanol: absence in rats, no tolerance and increased sensitivity in mice. Pharmacol Biochem Behav 24:1225-1228.

Mattingly BA, Gotsick JE (1989) Conditioning and experimental factors affecting the development of sensitization to apomorphine. Behav Neurosci 108:1311-1317.

Mattingly BA, Rowlett JK (1989) Effects of repeated apomorphine and haloperidol treatments on subsequent behavioral sensitivity to apomorphine. Pharmacol Biochem Behav 34:345-347.

Mattingly BA, Gotsick JE, Marin C (1988a) Locomotor activity and stereotypy in rats following repeated apomorphine treatments at 1-, 3-, or 7-day intervals. Pharmacol Biochem Behav 31:871-875.

Mattingly BA, Gotsick JE, Salamanca K (1988b) Latent sensitization to apomorphine following repeated low doses. Behav Neurosci 102: 553-558.

Mattingly BA, Rowlett JK, Graff JT, Hatton BJ (1991) Effects of selective D1 and D2 dopamine antagonists on the development of behavioral sensitization to apomorphine. Psychopharmacology (Berlin) 105:501-507.

McGeer PL, McGeer EG, Suzuki JS (1977) Aging and extrapyramidal function. Arch Neurol 34:33-35.

Morelli M, Di Chiara G (1987) Agonist-induced homologous and heterologous sensitization to D-1- and D-2-dependant contraversive turning. Eur J Pharmacol 141:101-107.

Morelli M, Fenu S, Di Chiara G (1987) Behavioral expression of D-1 receptor supersensitivity depends on previous stimulation of D-2 receptors. Life Sci 40:245-251.

Norman AB, Wyatt LM, Hildebrand JP, Kolmonpunporn M, Moody CA, Lehman MN, Sanberg PR (1990) Sensitization of rotation behavior in rats with unilateral 6-hydroxydopamine or kainic acid-induced striatal lesions. Pharmacol Biochem Behav 37:755-759.

Pei Q, Zetterstrom T, Fillenz M (1990) Tail pinch-induced changes in the turnover and release of dopamine and 5-hydroxytryptaminc in different brain regions of the rat. Neuroscience 35:133-138.

Phillips TJ, Dickenson S, Burkhart-Kasch S (1994) Behavioral sensitization to drug stimulant effects in C57BL/6J and DBA/2J inbred mice. Behav Neurosci 108:789-803.

Robinson TE, Jurson PA, Bennett JA, Bentgen KM (1988) Persistent sensitization of dopamine neurotransmission in ventral striatum (nucleus accumbens) produced by prior experience with (+)amphetamine: a microdialysis study in freely moving rats. Brain Res 462: $211-222$.

Rodriguez M, Castro R (1991) Apomorphine lowers dopamine synthesis for up to $48 \mathrm{~h}$ : implications for drug sensitization. Neuroreport 2:365-368.

Rouge-Pont F, Piazza PV, Kharouby M, Le Moul M, Simon H (1993) Higher and longer stress-induced increase in dopamine concentrations in the nucleus accumbens of animals predisposed to amphetamine self-administration. A microdialysis study. Brain Res 602:169-174.

Rowlett JK, Mattingly BA, Bardo MT (1991) Neurochemical and behavioral effects of acute and chronic treatment with apomorphine in rats. Neuropharmacology 30:191-197.

Rowlett JK, Mattingly BA, Bardo MT (1993) Neurochemical correlates of behavioral sensitization following repeated apomorphine treatment: assessment of the role of D1 dopamine receptor stimulation. Synapse 14:160-168.

Sanberg PR, Zoloty SA, Willis R, Ticarich CD, Rhoads K, Nagy RP, Mitchell SG, Laforest AR, Jenks JA, Harkabus LJ, Gurson DB, Finnefrock JA, Bednarik EJ (1987) Digiscan activity: automated measurement of thigmotactic and stereotypic behavior in rats. Pharmacol Biochem Behav 27:569-572.

Schallert T, Norton D, Razcok SE, Johnston RE, Becker JB (1994) Recovery of spontaneous behaviors after grafts of fetal ventral mesencephalic tissue into the dopamine-denervated rat striatum. Int Behav Neurosci Soc Abstr 3:78.

Segal DS, Kuczenski R (1987a) Behavioral and neurochemical characteristics of stimulant-induced augmentation. Psychopharmacol Bull 23:417-424.

Segal DS, Kuczenski R (1987b) Individual differences in responsiveness to single and repeated amphetamine administration: behavioral characteristics and neurochemical correlates. J Pharmacol Exp Ther 242:917-926.

Silverman PB, Ho BT (1981) Persistent behavioral effect of apomorphine in 6-hydroxydopamine lesioned rats. Nature 294:475-477.

Staunton DA, Wolfe BB, Groves PM, Molinoff PB (1981) Dopamine receptor changes following destruction of the nigrostriatal pathway: lack of a relationship to rotational behavior. Brain Res 211:315-327.

Stewart J, Vezina P (1988) Conditioning and behavioral sensitization. In: Sensitization in the nervous system (Kalivas PW, Barnes CD, eds), pp 207-224. Caldwell, NJ: Telford.

Sullivan RM, Fraser A, Szechtman H (1994) Asymmetrical orientation 
to edges of an openfield: modulation by striatal dopamine and relationship to motor asymmetries in the rat. Brain Res 637:114-118.

Tanaka T, Yokoo H, Mizoguchi K, Yosiha M, Tsuda A, Tanaka M (1991) Noradrenaline release in the rat amygdala is increased by stress: studies with intracerebral microdialysis. Brain Res 544:174176.

Ungerstedt U (1971a) Striatal dopamine release after amphetamine or nerve degeneration revealed by rotational behavior. Acta Physiol Scand 367:49-66.

Ungerstedt U (1971b) Post synaptic supersensitivity after 6 hydroxy- dopamine induced degeneration of the nigro-striatal dopamine system. Acta Physiol Scand 367:69-93.

Ungerstedt U, Arbuthnott GW (1970) Quantitative recording of rotational behavior in rats after 6-hydroxydopamine lesions of the nigrostriatal dopamine system. Brain Res 24:485-493.

Waddington J, Crow T (1979) Drug induced rotational behavior following unilateral intracerebral injection of saline-ascorbate solution: neurotoxicity of ascorbic acid and monoamine independent circling. Brain Res 161:371-376.

Wise RA (1988) Psychomotor stimulant properties of addiclive drugs. Ann NY Acad Sci 537:228-234. 